\title{
INFLUENCIA DEL CONSUMO DE BEBIDAS CARBONATADAS EN LA PREVALENCIA DE EROSIÓN DENTAL EN BASQUETBOLISTAS JUVENILES
}

\author{
INFLUENCE OF THE CONSUMPTION OF CARBONATED BEVERAGES IN THE \\ PREVALENCE OF DENTAL EROSION IN YOUNG BASKETBALL PLAYERS
}

\author{
Vladimir Lara A. ${ }^{1 a}$, Jarrín Maria José ${ }^{1 a}$, Toalombo Olivia ${ }^{1 a}$, Carrera Alex ${ }^{1 b}$, Dourado \\ Logersio Alessandro ${ }^{1 \mathrm{c}, 2 \mathrm{~d}}$, Armas Ana del Carmen ${ }^{1 \mathrm{~d}, \mathrm{e}}$
}

\section{RESUMEN}

Objetivo: Evaluar la prevalencia de erosión dental entre jugadores de baloncesto que refieren el consumo de bebidas carbonatadas. Materiales y métodos: se plantea un estudio transversal en una población de 60 basquetbolistas entre 8 a 18 años, que realicen actividad física más de 3 veces a la semana y que consuman bebidas energizantes más de 3 veces a la semana, sin síndromes o enfermedad sistémica ni discapacidad física o intelectual. Los participantes, fueron examinados clínicamente por un único examinador en cuanto a sus dientes anteriores superiores a través de índice de erosión dental BEWE y también por un cuestionario sobre conocimiento y consumo de bebidas ácidas. Resultados: se observó una alta prevalencia de erosión dental (56,6\%) en la población evaluada, el 41,67\% de participantes prefiere consumir bebidas hidratantes luego de la jornada de entrenamiento, con un alto porcentaje de bebidas carbonatadas al día; donde el 71,67\% conoce los efectos negativos del consumo de estas bebidas. Conclusión: La presencia de alteraciones en la estructura dental en la población evaluada es alta.

Palabras Clave: erosión dental; bebidas carbonatadas; Desgaste dental, desmineralización. (Fuente: DeCS BIREME)

\section{ABSTRACT}

Objective: To assess the prevalence of dental erosion among basketball players who report consumption of carbonated beverages. Materials and methods: a cross-sectional study is proposed in a population of 60 basketball players aged 8 to 18 years, who perform physical activity more than 3 times a week and who consume energy drinks more than 3 times a week, without syndromes or systemic disease neither physical or intellectual disability. The participants were examined clinically by a single examiner regarding their upper anterior teeth through BEWE tooth erosion index and also by a questionnaire on knowledge and consumption of acidic beverages. Results: a high prevalence of dental erosion was observed (56.6\%) in the evaluated population; $41.67 \%$ of participants preferred to consume hydrating drinks after the training day, with a high percen tage of carbonated beverages per day; where $71.67 \%$ know the negative effects of the consumption of these drinks. Conclusion: The presence of alterations in dental structure in the evaluated population is high.

Keywords: tooth erosion, carbonated beverages tooth wear, tooth hypo mineralization. (Source: MeSH NLM)

Recibido: 16 de julio de 2018

${ }^{1}$ Universidad Tecnológica Equinoccial. Ecuador

2 Universidad Estadual de Ponta Grossa- Brasil

${ }^{a}$ Estudiante. ${ }^{b}$ Odontólogo, ${ }^{c}$ PhD en Materiales Dentales, Docente invitado, ${ }^{d}$ Docente ${ }^{e} \mathrm{PhD}$ en Operatoria dental

Correspondencia:

Ana Luisa Vladimir

Correo electrónico: vladylara12_@hotmail.com
Aprobado: 20 de setiembre de 2018

Publicado: 30 de diciembre de 2018
Este es un artículo Open Access distribuido bajo la licencia Creative Commons Atribución-NoComercial- Compartir Igual 4.0

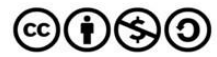

Citar como: Lara Vladimir A, Jarrín Maria José, Toalombo Olivia, Carrera Alex, Dourado Logersio Alessandro, Armas Ana. Influencia del consumo de bebidas carbonatadas en la prevalencia de erosión dental en basquetbolistas juveniles K IRU. 2018; 15(4): 166 - 170.

https://doi.org/10.24265/kiru.2018.v15n4.02 


\section{INTRODUCCIÓN}

L La erosión dental describe a los resultados físicos de la pérdida localizada, patológica y crónica de tejido dental duro siendo removido químicamente de la superficie sin envolver bacterias ${ }^{(1)}$. La erosión dental en los atletas es una preocupación creciente debido a un mayor interés por un estilo de vida saludable ${ }^{(2)}$, que implican ejercicio regular y dieta sana (3); así como el incremento en el consumo de bebidas deportivas que proporcionan carbohidratos, electrolitos y reemplazo de fluidos de uso frecuente entre los atletas antes de competir en eventos de larga distancia para aumentar los niveles de energía y durante la carrera para prevenir la deshidratación ${ }^{(4)}$.

El bajo $\mathrm{pH}$ de estas bebidas deportivas se ha observado aumenta el riesgo de erosión ${ }^{(5,6)}$, notándose un aumento en su consumo pese al asesoramiento dietético de un entrenador y nutricionista, influenciado por presiones comerciales existentes (7). La erosión dental es una pérdida de superficie del diente relacionada a un proceso químico que no involucra bacterias ${ }^{(8)}$. En los juegos olímpicos de verano de Londres 2012, se encontró que la erosión dental así como, caries y enfermedad periodontal, fueron las patologías más frecuentes de los deportistas ${ }^{\left({ }^{9}\right)}$.

Considerando su alta frecuencia reportada a nivel mundial y la falta de datos existentes en el Ecuador sobre el tema, este estudio busca evaluar la prevalencia de erosión dental en jugadores de baloncesto en una ciudad ubicada en la sierra central del Ecuador y relacionarlo con el consumo de bebidas carbonatadas.

\section{MATERIALES Y METODOS}

Se plantea un estudio transversal, en una población conformada por 60 participantes de 8 a 18 años basquetbolistas, que entrenan de manera constante en el coliseo central de San José de Chimbo provincia Bolívar, Ecuador. Previa aprobación de participación por parte de sus padres mediante firma del documento de consentimiento informado y verificación de cumplimiento de criterios de inclusión, consumir bebidas energizantes más de 3 veces a la semana, no presentar alteraciones dentales del tipo hipoplasia, fluorosis, restauraciones o lesiones cariosas con cavidad evidente; no presentar ningún tipo de enfermedad sistémica; síndrome o discapacidad; realizar actividad física más de 3 veces a la semana; realizar prácticas de higienización oral mínimo 2 veces al día.
En asamblea con presencia de padres y participantes fue socializado el proyecto, solicitándose llenar una encuesta considerado idóneo como elemento de recolección de información y fácil de aplicación, con objetividad en sus cuestionamientos y respuestas, con 12 preguntas con respuestas dicotómicas, sobre el conocimiento que el consumo de bebidas hidratantes y carbonatadas produce, así como la frecuencia de su consumo ${ }^{(10)}$. Cada ficha fue identificada con códigos para proteger la identidad de los participantes.

El examen clínico fue ejecutado por un único examinador con experiencia en la ejecución de este análisis quien empleó el índice de BEWE (11,12) empleado en muchos estudios previos analizando individuos en edades similares a las evaluadas en este estudio (13-16) razón por la cual fue elegido para ser empleado en este estudio, con la utilización de luz artificial (fronto luz), equipo de exploración básico (espejo bucal y explorador dental OMS), gasas y algodones.

Todas las superficies de sus dientes fueran examinadas con excepción de los terceros molares, considerando la severidad de la erosión por superficie en una escala de 0 a 3, de acuerdo con las siguientes descripciones: 0 no existe pérdida de superficie; 1 - pérdida inicial de la textura superficial; 2 - defecto evidente con pérdida de tejido duro es menos de $50 \%$ del área de superficie y; 3 - defecto evidente con pérdida de tejido duro en más de $50 \%$. Registrando únicamente la superficie con el valor más elevado, obteniendo la puntuación total BEWE, por sextante.

Los datos recolectados tanto del índice BEWE por participante como la valoración obtenida de la encuesta fue registrada en tablas Excel previamente elaboradas. Estos fueron analizados con la correlación lineal de Pearson mediante el programa SPSS.

\section{RESULTADOS}

El análisis descriptivo de los datos obtenidos permitió determinar que, en relación al consumo de bebidas hidratantes, $41,67 \%$ de participantes prefiere consumir bebidas hidratantes luego de la jornada de entrenamiento, en una media de 2 botellas al día $33,33 \%$ una botella al día, $16,67 \%$ tres botellas al día y un $8,33 \%$ más de tres botellas al día (Tabla. 1 ).

Considerando el incremento o disminución del consumo de bebidas carbonatadas y la actual actividad física, los participantes refirieron un incremento en el consumo 
(76,67\% (Tabla. 2.). El $71,67 \%$ refirió conocer sobre los efectos negativos de su consumo y un $75 \%$ relato el haber experimentado efectos negativos tras su consumo. Un $83,33 \%$ de participantes afirmo conocer sobre las bebidas carbonatadas sin azúcar, siendo el consumo directo de la botella el modo preferido por los participantes, en un $75 \%$.

Tabla 1. Cantidad de veces al día de consumo de refrescos

\begin{tabular}{lcl}
\hline Alternativa & Frecuencia & Porcentaje \\
\hline Una sola vez & 10 & 16.67 \\
Dos veces & 25 & 41.67 \\
Tres veces & 15 & 25.00 \\
Más de tres & 10 & 16.67 \\
Total & 60 & 100.00 \\
\hline
\end{tabular}

Tabla 2. Consumo de las bebidas carbonatadas por los participantes

\begin{tabular}{clc}
\hline Alternativa & Frecuencia & Porcentaje \\
\hline Incrementado & 48 & 76.77 \\
Disminuido & 14 & 23.33 \\
Total & 60 & 100.00 \\
\hline
\end{tabular}

El examen clínico revelo que $5 \%$ de los participantes no presentaron desgaste alguno en la superficie de sus dientes, $28,33 \%$ ligero desgaste; $41,67 \%$ con menos del $50 \%$ de superficie, y $15 \%$ con desgaste de más del $50 \%$.

Para poder determinar el grado de relación entre la encuesta aplicada y la observación clínica, considerando el índice BEWE, se aplicó un análisis de correlación simple, encontrando una relación estadísticamente significativa entre la erosión presentada en los deportistas y la frecuencia de consumo de estas bebidas carbonatadas $(p<0,004)$.

\section{DISCUSIÓN}

Los resultados evidenciaron que el consumo de bebidas carbonatadas que consume la población evaluada es alta, en general al menos una botella al día; en relación a la presencia de lesiones erosivas, cerca de $56,6 \%$ de la población evaluada presentó erosión dental, explicado por el consumo de diferentes bebidas energéticas comercialmente disponibles en el medio, generalmente con $\mathrm{pH}$ ácido, asociado a la presencia de gas carbónico en su composición, lo que las convierte en sustancias potencialmente desmineralizantes ${ }^{(17)}$ y por tanto erosivas ${ }^{(18,19)}$.

Frente a un $\mathrm{pH}$ de 2.4 de las bebidas carbonatadas, se produce una acelerada liberación de iones de calcio y fosfato desde el esmalte hacia el medio circundante ${ }^{(20)}$ el ambiente bucal si bien es capaz de recuperar su pH neutro natural después de la ingestión de sustancias ácidas, el acelerado y pronunciado descenso no consigue ser cubierto por la saliva, afectando la superficie dental ${ }^{(21)}$ constituyéndose la erosión en un proceso destructivo crónico (22) contrapuesto a la acción ácida producida por bacterias desencadenantes de la caries dental ${ }^{(23)}$.

Estudios ejecutados en poblaciones en edades similares, revelan una elevada prevalencia de la erosión ${ }^{(2,5)}$, asociándola a una etiología multifactorial ${ }^{(12)}$, donde la dieta (24), el reflujo gastroesofágico ${ }^{(19)}$, el tiempo empleado durante la competencia y su preparación ${ }^{(9)}$ adquieren gran importancia.

Si bien una de las limitaciones que el estudio presentó fue la reducida muestra, los resultados nos permiten tener una idea clara del problema que el consumo de bebidas carbonatadas en la población que ejecuta actividad física desencadena (11), a esto se suma el hecho de contar con la composición de estas, con azúcares que en un determinado momento desencadenarían la presencia de caries dental asociada a su acúmulo, que si bien no fue evaluada en este estudio puede desencadenar nuevos análisis ${ }^{(25)}$, sobre todo al considerar que en la cultura ecuatoriana el consumo de alimentos ácidos y azucarados es frecuente lo que puede desencadenar daño irremediable a nivel del esmalte dental (26).

La población deportista es altamente susceptible a diferentes trastornos por la exigencia física que su ejecución representa (14) desencadenando un mayor consumo de carbohidratos en la dieta ${ }^{(27)}$, asociada a la práctica de actividad física anaerobia realizada con la perdida de electrolitos y deshidratación ${ }^{(28)}$ que en el organismo se produce.

Como odontólogos estamos en la obligación de plantearnos estrategias preventivas de concientización entre padres, educadores y jóvenes de centros deportivos sobre los efectos del consumo de bebidas carbonatadas ${ }^{(29,30)}$ tanto a nivel dental como sistémico.

Se concluye que la presencia de alteraciones en la estructura dental en la población evaluada es alta. 
Nuevos estudios requieren ser ejecutados para poder establecer relación con el consumo de bebidas carbonatas.

Contribuciones de autoría: LV, JM, TO, CA, DL, AA Contribuyeron en el diseño del estudio, recopilación de datos y análisis estadístico. Todos los autores aprobaron la versión final del artículo.

Fuente de financiamiento: El estudio fue financiado por los autores.

Conflicto de intereses: Los autores declararon no tener conflicto de interés en este artículo.

\section{REFERENCIAS}

1. Torres D, Fuentes R, Bornhardt T. Erosión dental y sus posibles factores de riesgo en niños: revisión de la literatura. Rev Clin Periodoncia Implantol Rehabil Oral. 2016; 9(1): 19-24.

2. Sirimaharaj V, Brearley Messer L, Morgan MV. Acidic diet and dental erosion among athletes. Aust Dent J. 2002 Sep; 47 (3): 228-36.

3. Tiwari Vidhatri, Saxena Vrinda, Tiwari Utkarsh, Singh Aishwarya, Santha Binu, Diwedi Vartul, Jain Manish. Assessment of dental erosion among sports personnel in central India. Saudi J Sports Med. 2015; 15(3):226-230

4. Lussi A, Ganss C. Erosive Tooth Wear. Monogr Oral Sci. Basel, Karger, 2014; 25: 22-31

5. Needleman I, Ashley P, Petrie A, Fortuna F, Turner $W$, Jones $\mathrm{J}$, et al. Oral health and impact on performance of athletes participating in the London 2012 Olympic Games: a cross-sectional study. Br J Sports Med. 2013 Nov; 47 (16): 1054-8.

6. Habib M, Hottel TL, Hong L.Prevalence and risk factors of dental erosion in American children. J Clin Pediatr Dent 2013; 38 :143-8

7. Ehlen LA, Marshall TA, Qian F, Wefel JS, Warren JJ. Acidic beverages increase the risk of in vitro tooth erosion. Nutr Res. 2008 May; 28(5):299-303.

8. Ostrowska A, Szymański W, Kołodziejczyk Ł, Bołtacz-Rzepkowska E. Evaluation of the Erosive Potential of Selected Isotonic Drinks: In Vitro Studies. Adv Clin Exp Med. 2016 Nov-Dec; 25(6):1313-1319.

9. Kumar D, Acharya S, Mishra P, Debnath N, Vasthare R. Prevalence and risk factors for dental erosion among 11 to 14 years old school children in South India. J Oral Science 2013; 55(4):329-36

10. Holbrook WP, Arnadottir lb, Hlöoversson SO, Arnarsdottir E, Jonsson Sh, Saemundsson SR.The basic erosive wear examination (BEWE) applied retrospectively two studies. Clin Oral Invest 2013 Dec;18(6):1625-9.
11. Fresno MC, Angel P, Arias R, Muñoz A. Grado de acidez y potencial erosivo de las bebidas energizantes disponibles en Chile. Rev. Clin. Periodoncia Implantol. Rehabil. Oral. 2014 abril; 7(1): 5-7.

12. Salas MM, Nascimento GG, Vargas-Ferreira F, Tarquinio SB, Huysmans MC, Demarco FF. Diet influenced tooth erosion prevalence in children and adolescents: Results of a meta-analysis and meta-regression. J Dent. 2015 Aug; 43(8):865-75. doi: 10.1016/j.jdent.2015.05.012. Epub 2015 Jun 7. Review. PubMed PMID: 26057086

13. Sovik JB, Skudutyte-Rysstad R, Tveit AB, Sandvik L, Mulic A. Sour sweets and acidic beverage consumption are risk indicators for dental erosion. Rev Caries Research. 2015; 9(1)

14. Gupta R, Solanki A, Sharma S, Gumber P, Sharma A, Upadhyay R. A Knowledge, Attitude and Practices of Soft Drinks among Adolescent Students and their Dental Health: A Questionnaire Study. Int J Dent Health Concern 2015;1:1-5

15. Wiegand A, Müller J, Werner C, Attin T. Prevalence of erosive tooth wear and associated risk factors in 2-7-year-old German kindergarten children. Oral Dis. 2006; 12(2):117-24

16. Muller-Bolla M, Courson F, Smail-Faugeron V, Bernardin T, Lupi-Pégurier L. Dental erosion in French adolescents. BMC Oral Health. 2015 Nov 19;15:147.

17. Alaraudanjoki V, Laitala M-L, Tjäderhane L, Pesonen P, Lussi A, Anttonen V. Association of erosive tooth wear and dental caries in Northern Finland Birth Cohort 1966 - an epidemiological cross-sectional study. BMC Oral Health. 2017; 17: 6

18. Hamasha AA, Zawaideh FI, Al-Hadithy RT. Risk indicators associated with dental erosion among Jordanian school children aged 12-14 years of age. Int J Paediatr Dent. 2014 Jan;24(1):56-68. doi: 10.1111/ipd.12026. Epub 2013 Feb 24. PubMed PMID: 23432693.

19. Ab Halim N, Esa R, Chew HP. General and erosive tooth wear of 16-year-old adolescents in Kuantan, Malaysia: prevalence and association with dental caries. BMC Oral Health. 2018; 18

20. Antunes LS, Veiga L, Nery VS, Nery CC, Antunes LA. Consumo de bebidas deportivas y la erosión dental entre los corredores aficionados. J Oral Sci. 2017; 59 (4): 639-643.

21. Zebrauskas A, Birskute R, Maciulskiene V. Prevalence of Dental Erosion among the Young Regular Swimmers in Kaunas, Lithuania. J Oral Maxillofac Res. 2014 Jul 1;5(2):e6..

22. Barbero Fernández LA, Benito Vicente MC, Martín Carreras-Presas C, Gutiérrez Cardenas L, Barbero Palao A. La salud bucal en deportistas: puesta al día. Cient. Dent. 2017; 14; 2; 87-91 
23. Calatrava L. Índices Epidemiológicos Del Desgaste Dental Erosivo.Revision Bibliográfica. Revista de Operatoria dental y biomateriales.2015; 4(2): 32-38

24. Joshi M, Joshi N, Kathariya R, Angadi P, Raikar S. Techniques to Evaluate Dental Erosion: A Systematic Review of Literature. J Clin Diagn Res. 2016 Oct, 10(10):1-7

25. Kitchens M, Owens BM. Effect of carbonated beverages, coffee, sports and high energy drinks, and bottled water on the in vitro erosion characteristics of dental enamel. J Clin Pediatr Dent. 2007 Spring;31(3):153-9. PubMed PMID: 17550037. 